\title{
EEG Alpha Power and Coherence time courses in a Sustained Attention Task
}

\begin{abstract}
Alpha activity at certain brain regions and the functional relationships between these regions quantified by alpha coherence were examined in a fixed sequence Sustained Attention to Response Task (SART), which involves the withholding of key presses to rare targets. The effects of distinguishing smaller sub bands within the alpha band to explain distinct cognitive components are assessed. It was found that in two of three subjects average power in the alpha1 band was phasically related to stimulus presentation, possibly reflecting short-term attentional processes. Also in two of the three subjects, average power in the alpha-2 band showed a relatively slow desynchronisation approaching the rare target, possibly reflecting expectancy.
\end{abstract}

Keywords - Alpha activity, EEG coherence

\section{INTRODUCTION}

Electroencephalogram (EEG) power in the alpha frequency range $(7.5-12.5 \mathrm{~Hz})$ has long and widely been regarded as a physiological correlate of wakefulness in humans. The alpha rhythm, usually defined by its distribution in the posterior scalp region and its reactivity to eye-opening/closing and alerting, may be seen as a peak in the EEG frequency spectrum within the alpha range [1]. In [2], alpha activity is proposed to be related to cognitive and memory performance and brain maturity.

The long-term changes in alpha power (such as changes with age), termed tonic changes are regarded distinctly from event-related or phasic changes measurable during task performance over short time periods. Alpha desynchronisation (suppression) is a well known phasic response to eye-opening and attentional processes. In [2] and [3] the alpha band (roughly $6.5-12.5 \mathrm{~Hz}$ ) is broken down into upper $(10.5-12.5 \mathrm{~Hz})$, and lower $(6.5-10.5 \mathrm{~Hz})$ alpha bands shown to selectively reflect sensory-semantic, and attentional processes respectively. The lower band is further subdivided into alpha- 1 and alpha- 2 , pertaining to general attention and expectancy respectively.

In this paper the authors investigate the relatedness of alpha power to the attentional demands of a Sustained Attention to Response Task (SART) within both the overall alpha band and alpha subbands. Also investigated is the spectral relatedness of alpha activity at different electrode sites, as measured by coherence.

Sustained attention is a psychological construct that describes the readiness of a subject to detect or respond to rarely occurring signals over prolonged periods of time. The SART task is intended to specifically tax intrinsic, endogenously maintained attention as distinct from exogenously controlled alertness, which is governed by such factors as novelty, salience and stimulus change [4]. Imaging studies have demonstrated that activation of frontal and parietal cortical areas, mostly in the right hemisphere are associated with sustained attention performance $[5,6]$. This paper thus focuses on the consistently documented frontal-parietal attention network by choosing appropriate scalp locations and also investigates right hemisphere bias.

Coherence measures are normalized spectral crosscorrelations, given by

$$
C_{x y}(f)=\frac{S_{x y}(f)}{\sqrt{S_{x x}(f) S_{y y}(f)}}
$$

where $S_{x x}(f)$ and $S_{y y}(f)$ are auto-power spectral densities of two stationary processes $x$ and $y$, and $S_{x y}(f)$ is their cross-power spectrum. Coherence analysis has been used extensively to study the spatial and temporal structure of EEGs, particularly functional coupling between cortical structures and oscillatory information propagation during different tasks, e.g. finger movement [7] and word processing [8]. The results of the coherence study in [8] in particular supports previous findings that the alpha-1 band predominately reflects sensory processes and attention processes.

\section{METHODOLOGY}

\section{A. The Task}

30-channel EEG was recorded during a fixed SART paradigm [4]. In this procedure a series of numbers " 1 " to "9" are presented in sequential order and subjects were required to respond by button press to every number except the number " 3 ". Each digit was presented for $250 \mathrm{msec}$, followed by a 900 msec mask, giving a total trial length of 1150 msec. 105 sequences of " 1 " through "9" were presented, totaling 945 single digit presentations over an 18 minute period. Data were recorded in $\mathrm{AC}$ mode (gain: 500; band pass: $0.15-30 \mathrm{~Hz}$ ) and the A/D conversion rate was $1000 \mathrm{~Hz}$. Skin-Electrode junction impedances were kept as much as possible below $5 \mathrm{k} \Omega$.

\section{B. Subjects}

The data of ten subjects, of whom five had sustained traumatic brain injury (TBI), were examined visually for blink artifacts and abnormalities such as those caused by 
skin-electrode impedance fluctuations. The data of three normal subjects of ages 31,33 and 45 contained sufficiently little blinking to be included in the analysis.

\section{The Data}

Preceding the SART task, three 1-minute blocks of relaxed eyes-open data were recorded for each subject with one minute break in between. This facilitates comparison between results obtained during the task and a normative benchmark.

The importance of stringent omission of blinkcontaminated data lies in the fact that blinks typically have durations of the order of $100 \mathrm{msec}$, making it very possible for a blink artifact to masquerade as a $\sim 10 \mathrm{~Hz}$ component in spectral analysis by Fourier methods. Artifact rejection guards against the possibility of finding false stimulusrelated alpha power or coherence which is actually due to blinks which occur on presentation of every stimulus.

Electrode sites used in spectral analysis were frontal F3, FZ, F4, fronto-central FC3, FC4, FCZ, centro-parietal CP3, $\mathrm{CPZ}, \mathrm{CP} 4$, and parietal P3, PZ, P4 according to the international 10-20 system of electrode placement [9]. Interhemispheric (F3-F4, FC3-FC4, CP3-CP4, P3-P4) and intrahemispheric left (F3-CP3, F3-P3, FC3-CP3, FC3-P3) and right (F4-CP4, F4-P4, FC4-CP4, FC4-P4) pair-wise coherences were calculated within the band $0-25 \mathrm{~Hz}$ for eyes open data and SART task data separately.

\section{Power and Coherence Estimation Procedure}

The 18-minute-long subject data were epoched from the presentation of " 6 " to " 5 ", resulting in blocks of approximately 12 seconds. Only blocks in which no commission errors or ommision errors were made were retained. After visual inspection, $N=39$ artifact-free blocks were retained for subject $1, N=47$ for subject 2 and $N=53$ for subject 3 .

In this study a 1 -sec data segment was regarded as short enough to be locally stationary. This corresponds to a frequency resolution of $1 \mathrm{~Hz}$ in the following Short-Time Fourier analysis: Each segment was first Hanning windowed to reduce spectral leakage effects, and its Discrete Fourier Transform (DFT) was then calculated to give complexvalued quantity $X_{i}^{n}(k)$ for segment $i$ in block $n$ of channel $x$. Estimates of auto-spectra were calculated by averaging over the $N$ blocks:

$$
\hat{S}_{x x, i}(k)=\frac{1}{N} \sum_{n=1}^{N}\left|X_{i}^{n}(k)\right|^{2}
$$

Similarly, cross-spectra between channel $x$ and $y$ were calculated as:

$$
\hat{S}_{x y, i}(k)=\frac{1}{N} \sum_{n=1}^{N} X_{i}^{n}(k) \overline{Y_{i}^{n}(k)}
$$

where bar denotes complex conjugation. Magnitude-squared Coherence was then calculated using these estimates in (1):

$$
C_{x y, i}^{2}(k)=\frac{\left|\hat{S}_{x y, i}(k)\right|^{2}}{\hat{S}_{x x, i}(k) \hat{S}_{y y, i}(k)}
$$

This function provides a bounded and normative measure of association, taking on values between 0 and 1 , with 0 in the case of independence and 1 in the case of a perfect linear relationship.

To obtain a value of coherence over a certain frequency band, band-averaged coherence is used:

$$
C_{x y, i}^{2}\left(k_{1}, k_{2}\right)=\frac{\left|\sum_{k=k_{1}}^{k_{2}} \hat{S}_{x y, i}(k)\right|^{2}}{\sum_{k=k_{1}}^{k_{2}} \hat{S}_{x x, i}(k) \sum_{k=k_{1}}^{k_{2}} \hat{S}_{y y, i}(k)}
$$

The bands used in the analysis were the entire alpha band (7-12 Hz), and subbands defined therein, alpha-1 (7-8 $\mathrm{Hz}$ ), alpha-2 $(9-10 \mathrm{~Hz})$ and upper-alpha $(11-12 \mathrm{~Hz})$.

Time-courses of power and coherence in these bands were obtained by shifting the segment in steps of $125 \mathrm{msec}$ from the start to the end of the block, using (5) at each step. This resulted in 92 segments per block. The time index for the $i$ th power or coherence value was taken as the midpoint of segment $i$.

For the eyes-open data 120 disjoint segments were used, 40 from each of the three minute-long blocks, and power and coherence estimates were calculated from averages across these segments.

In each band the power relative to the corresponding eyes-open reference band power was calculated rather than absolute power. This is important when comparing across subjects, as acquisition conditions may vary across sessions. Coherence was not adjusted, but is compared to eyes-open coherence in the results.

\section{E. Coherence Confidence Limits}

For a finite amount of data, estimations of coherence will have some associated error. It is therefore useful to quantify the variability of the estimate in the form of a confidence interval so that the significance of interesting features may be assessed. This confidence interval depends on the number of disjoint segments used for the estimate, $L$. First a variance stabilizing transform - arctanh of the modulus of coherence - is used to produce a distribution closer to normal. An expression for the variance of this value,

$$
\operatorname{var}\left\{\operatorname{Tanh}^{-1}\left|C_{x y}(f)\right|\right\}=\frac{1}{2 L},
$$

leads to approximate $95 \%$ confidence limits of

$$
\operatorname{Tanh}^{-1}\left|C_{x y}(f)\right| \pm 1.96 / \sqrt{2 L},
$$


which are transformed back to the domain of $\left|C_{x y}(f)\right|^{2}$ [10]. It may also be necessary to include in a coherence plot the value

$$
1-(0.05)^{1 /(L-1)}
$$

as an estimate of the upper $95 \%$ confidence limit under the hypothesis of independent $\mathrm{x}$ and $\mathrm{y}$. All of the above confidence limit estimation principles were applied for both eyes-open and task coherence.

\section{RESULTS}

\section{A. Power}

In all three cases it was found that certain features were more pronounced in one of the subbands than in the entire alpha range. Fig.1 shows a plot of relative power at electrode site PZ for Subject 2. A clear divergence of time course can be seen between 8 and $10 \mathrm{~Hz}$. This suggests that care should be taken when examining broad frequency ranges.

According to [2], power should reflect general attentional processes, while alpha-2 should reflect expectancy. Fig.2(a) and (b) show the alpha-1 power time courses for Subjects 1 and 3 respectively. In both cases alpha-1 is seen to respond phasically to stimulus presentation with desynchronisations prior to each of the nine stimuli. This effect was by far most prominent in the right parietal area. Subject 2 also demonstrated a phasic response to stimuli, but was more prominent in the alpha-2 range (fig.2(c)). Also in this band, a slower baseline suppression may be seen after presentation of "1". This effect is distributed bilaterally in the parietal region. Subject 1 shows a similar alpha- 2 baseline drop coming up to the " 3 " (fig.2(d)), the response being earlier in this case.

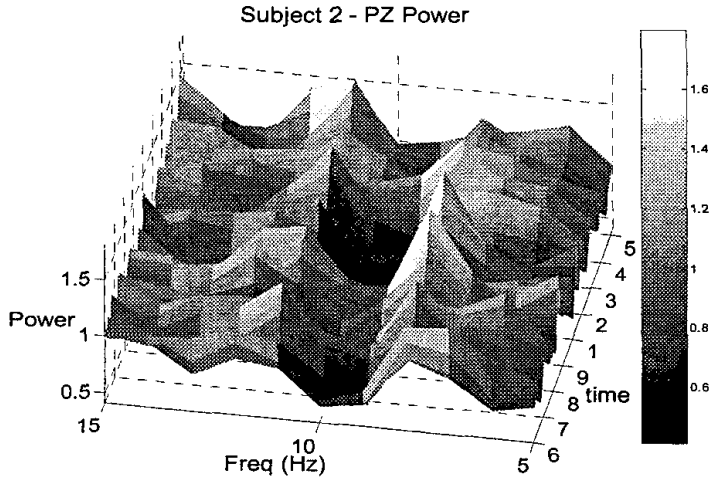

Fig. 1: Relative power (amplitude squared) at PZ (5-15Hz) for subject 2. Marked on the time axis is the sequence of digits presented.

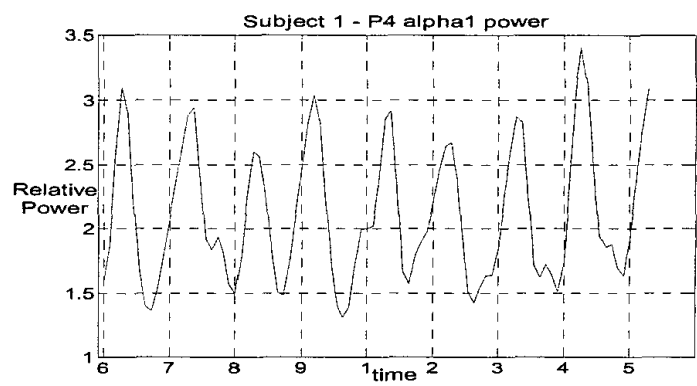

(a)

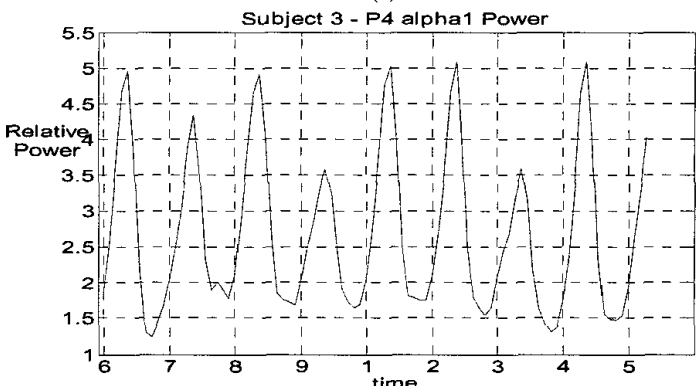

(b)

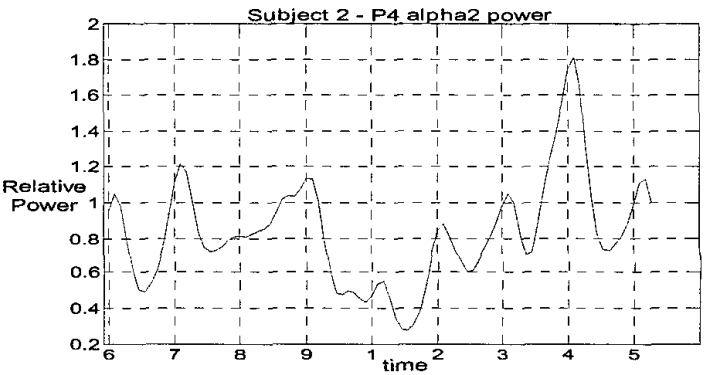

(c)

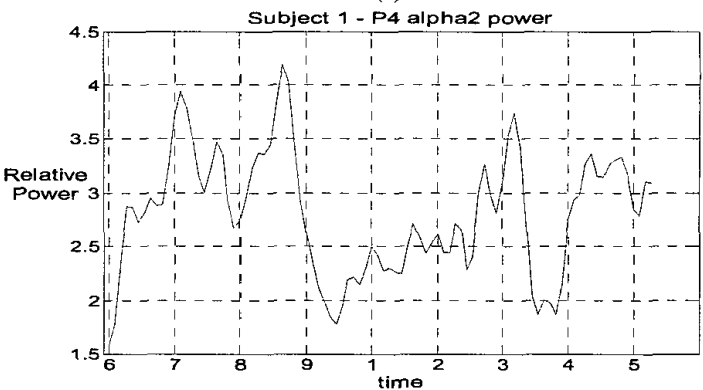

(d)

Fig.2: band power time courses at right parietal P4

\section{B. Coherence}

Fig.3 shows intrahemispheric coherence between right hemisphere locations F4 and CP4 in the alpha-2 range for subject 2. Significance of coherence estimates were assessed using the $95 \%$ confidence limits derived above. A peak can be seen just before the presentation of a " 3 ". 


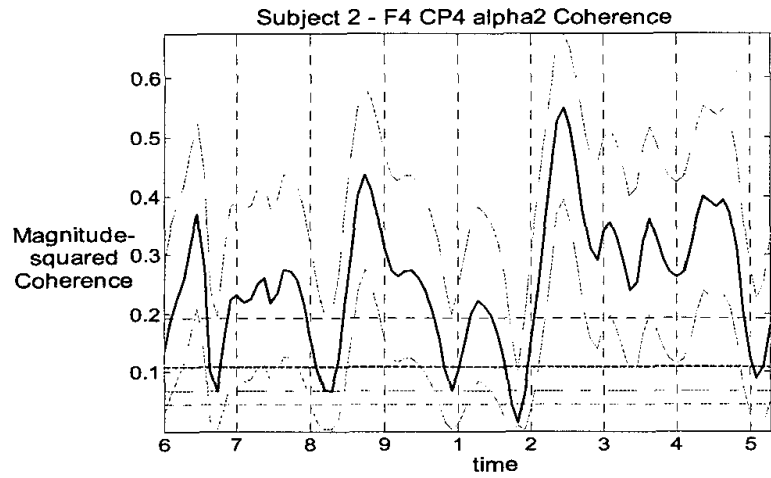

Fig.3: Alpha-2 time course of intrahemispheric coherence between F4 and CP4 for subject 2 . The dashed traces above and below the coherence plot represent the upper and lower confidence limits respectively. The horizontal dashed line represents eyes-open coherence, with confidence limits, and the horizontal dash-dot line represents the confidence limit assuming independence, as given by ( 8 ).

\section{DISCUSSION}

In the lower alpha-1 frequency band peaks and troughs in relative power were found which respond synchronously with stimulus presentation. Although the data were screened so that no blinks were present, there is still the possibility that this phasic responding of alpha- 1 in the parietal region is due to visual evoked potential effects.

In the lower alpha-2 band there is some evidence of expectancy effects, though in one of the two subjects exhibiting this feature, subject 1 , additional periods of alpha-2 suppression were found in the averaged sequence which suggest a more erratic time course of an expectancy correlate than might be expected.

Coherence between frontal and centro-parietal right hemisphere regions for one subject shows increased synchronization prior to the no-go stimulus. Although no evidence of this peak could be seen on the left side suggesting hemispheric bias, the conclusiveness of this feature is limited by the absence of concurrence in subjects 1 and 3 .

An important shortcoming of the data is the number of uncontaminated blocks used to estimate coherence. In this study the number of blocks used ranged from 39 to 53, whereas in other coherence studies [7,11], approximately 150 are used. Obviously the longer the blocks used, the more probable the artifact contamination.

As regards the paradigm used, though the fixed sequence distinguishes cognitive features such as expectancy, more distinct features in coherence time courses may result from randomizing digit presentation, as in the random SART [4]. In this case Go/No-Go comparisons may provide better insight to synchronization phenomena involved in the motor inhibition process.

Other time-frequency analysis methods may provide clearer results. For band power analysis an alternative to the methods presented here is the Induced Band Power measure
[2]. Also for both power and coherence analysis, autoregressive modelling and wavelet analysis may be used.

\section{CONCLUSION}

Evidence was presented here which supports previously documented EEG activity reflecting phasic attention and expectancy processes during sustained attention tasks. Also, the results would encourage division of the alpha band into smaller sub-bands to distinguish cognitive components.

A possible application of this work would be to use the features presented here in a feedback paradigm for assessment or improvement of reduced attentional capacity implicated in syndromes such as attention deficit hyperactivity disorder.

\section{REFERENCES}

[1] Bruce J. Fisch, EEG Primer: basic principles of digital and analog EEG, 3 rd ed., Elsevier, 1999, pp.185-190.

[2] W. Klimesch, "EEG alpha and theta oscillations reflect cognitive and memory performance: a review and analysis," Brain Res. Rev., 29 (1999), pp. 169-195.

[3] W.Klimesch, M. Doppelmayr, H. Russegger, T. Pachinger, J. Schwaiger, "Induced alpha band power changes in the human EEG and attention", Neurosci. Lett., 244 (1998), pp. 73-76.

[4] 1.H. Robertson, T. Manly, J. Andrade, B.T. Baddeley, J. Yiend, "'Oops!': Performance correlates of everyday attentional failures in traumatic brain injured and normal subjects," Neuropsychologia, Vol. 35, No. 6, 747-758, 1997. [5] M.I. Posner, S.E. Petersen, "The attention system of the human brain," Annu.Rev. Neurosci., 13 (1990), pp. 25-42.

[6] M. Sarter, B. Givens, J.P. Bruno, "The cognitive neuroscience of sustained attention: where top-down meets bottom-up," Brain Res. Rev., 35 (2001), pp. 146-160.

[7] C. Andrew, G Pfurtscheller, "Event-related coherence as a tool for studying dynamic interaction of brain regions", Electroenceph. Clin. Neurophysiol., 98 (1996) pp. 144-148.

[8] B. Schack, P. Rappelsberger, C. Anders, S. Weiss, E. Moller, "Quantification of synchronization processes by coherence and phase and its application in analysis of electrophysiological signals," Int. Jour. Bif. Chaos, Vol. 10, No. 11 (2000), pp. 2565-2586.

[9] American Electroencephalographic Society "Guidelines for Standard Electrode Position Nomenclature," J. Clin. Neurophysiol., 8(2): 200-202, (1991).

[10] D. M. Halliday, J. R. Rosenburg, A. M. Amjad, P. Breeze, B. A. Conway, S. F. Farmer, "A framework for the analysis of mixed time series/point process data - theory and application to the study of physiological tremor, single motor unit discharges and electromyograms", Prog. Biophys. Molec. Biol., vol 64, no. 2/3, pp. 237-278, 1995.

[11] T. Shibata et al, "The time course of interhemispheric EEG coherence during a GO/NO-GO task in humans," Neurosci. Lett. 233 (1997), pp. 117-120. 